\title{
Th17/1-Biased Inflammatory Environment Involved in the Response of Epithelial Cells to Antigen Stimuli in Nasal Polyps
}

\author{
Guangfu Xu $\mathbb{D}^{1}$, Silong Chen $\mathbb{D}^{1},{ }^{1}$ Yuchun Dong $\mathbb{D}^{2},{ }^{2}$ and Li Xiao $\mathbb{D}^{1}$ \\ ${ }^{1}$ Medical College of China Three Gorges University, China \\ ${ }^{2}$ The Department of Otolaryngology, ChangYang People's Hospital, China \\ Correspondence should be addressed to Li Xiao; xiaoli-cn@163.com
}

Received 23 February 2021; Accepted 24 May 2021; Published 10 June 2021

Academic Editor: Patrice Petit

Copyright (C) 2021 Guangfu Xu et al. This is an open access article distributed under the Creative Commons Attribution License, which permits unrestricted use, distribution, and reproduction in any medium, provided the original work is properly cited.

\begin{abstract}
Several studies showed that IL-17A was significantly increased in nasal polyps (NPs). However, the source and characteristics of IL17A-producing cells in NPs were not fully understood. We isolated mononuclear cells from NPs and uncinate tissues and analyzed them using flow cytometry. The results indicated that IL-17A was increased in NP tissues compared to uncinate tissues. The main IL-17A-expressing cells were $\mathrm{CD}^{+} \mathrm{T}$ cells in NP tissues, including Th17 cells, Tc17 cells, and $\gamma \delta \mathrm{T} 17$ cells. Not similar to those in uncinate tissues, the majority of Th17 cells highly coexpressed IFN- $\gamma$ in NP tissues, such as Th17/1 cells, which highly expressed CXCR3, CCR6, ROR $\gamma$ t, and T-bet. Furthermore, Th17/1-biased environment increased the response of nasal epithelial cells to bacterial and viral stimuli, implying that Th17/1 cells play a greater role in the pathological development of NPs than Th17 or Th1 cells.
\end{abstract}

\section{Introduction}

Nasal polyps (NPs) are a chronic inflammatory disease of the upper airway with impaired quality of life which affects from $1 \%$ to $4 \%$ of the population [1]. Patients with NPs typically present with nasal obstruction, rhinorrhea or hyposmia, and so on. It is still one of the most challenging diseases in clinical rhinology because of its complex etiology and frequent recurrence [2]. Although the etiology and pathogenesis of NP have not been fully elucidated, it is probably a multifactorial disease with several different etiological factors in which bacteria, viruses, and fungi are closely related to the establishment of the inflammatory process [3]. Our earlier [4] and other studies [5] have demonstrated that different kinds of immune cells and a complicated cytokine response network were involved in the pathogenesis of immunemediated disorders in NP tissues.

In recent years, the role of IL-17A in respiratory diseases has intensively been investigated. It was consensus that the expression of IL-17A was significantly upregulated in NP tissues [6] and significantly positively correlated with clinical features [7]. IL-17A may be involved in the development and remodeling of NPs through its local immune modulation [8]. However, the source and phenotype of IL-17A-producing cells in NP tissues are still not clear. In this study, we performed single-cell analysis to identify the IL-17A-producing cells and examined whether a Th17/1-biased cytokine environment regulated the response of nasal epithelial cells to viral and bacterial infections.

\section{Materials and Methods}

2.1. Patients. NP and uncinate tissues were recruited from ChangYang People's Hospital. 35 NP samples were obtained during routine functional endoscopic sinus surgery. The diagnosis was made according to the criteria of the European Position Paper on Rhinosinusitis and Nasal Polyps 2007 (EP3OS 2007) [9] and was based on patient history, clinical examination, nasal endoscopy, and sinus computed tomography (CT) scanning. For details of subjects' characteristics, 
see Table 1. 16 uncinate tissue samples were recruited from patients undergoing septoplasty during septal surgery. Patients with established immunodeficiency, pregnancy, diagnosis of classic allergic fungal sinusitis, or cystic fibrosis were excluded from the study. None of the subjects used oral or intranasal steroids for at least 2 weeks before sample collection. All subjects signed informed consent forms, and the study was approved by the local ethics committees of participating hospitals.

2.2. Cell Isolation and Tissue Homogenate. Tissue samples were cut into small pieces, digested in incomplete RPMI1640 with endotoxin-free collagenase I $(2 \mathrm{mg} / \mathrm{ml}$, SigmaAldrich, St Louis, MO, USA) for $30 \mathrm{~min}$ at $37^{\circ} \mathrm{C}$. The digested fragments were filtered through a $100 \mathrm{~mm}$ cell nylon mesh (BD Bioscience PharMingen, San Diego, CA, USA) to prepare a single-cell suspension. The mononuclear cells from NP and uncinate tissues were obtained by Ficoll-Hypaque (Tianjin Hao Yang Biological Manufacture, Tianjin, China) density gradient centrifugation.

Fresh tissue specimens were homogenized on ice for 3 min with PBS ( $1 \mathrm{ml}$ of PBS/100 $\mathrm{mg}$ of tissue) containing a protease inhibitor cocktail (Keygentec, Nanjing, Jiangsu, China). The homogenates were then centrifuged at 4000 $\mathrm{rpm}$ for 20 minutes at $4^{\circ} \mathrm{C}$, and the supernatants were stored at $-80^{\circ} \mathrm{C}$ for ELISA.

2.3. Flow Cytometry. Cells were firstly incubated with red fluorescent reactive dye (live/dead fixable dead cell stain kit, Invitrogen) for 30 minutes for dead cell discrimination. Then, the cells were resuspended with PBS buffer containing $0.1 \%$ BSA and $0.05 \%$ sodium azide. For surface staining, cells were incubated with the respective mAbs at $4^{\circ} \mathrm{C}$ in the dark for $30 \mathrm{~min}$. For the detection of intracellular cytokines, cells were fixed with $4 \%$ paraformaldehyde and permeabilized in PBS buffer containing $0.1 \%$ saponin (SigmaAldrich), $0.1 \% \mathrm{BSA}$, and $0.05 \% \mathrm{NaN}_{3}$ for at least $2 \mathrm{~h}$ at $4{ }^{\circ} \mathrm{C}$ and then stained with conjugated mAbs. For the detection of intracellular transcription factors, cells were stained for surface antigens, followed by fixation, permeabilization with Permeabilization/Fixation buffer (BD Bioscience PharMingen), and staining according to the protocol of the Permeabilization/Fixation Kit. Stained cells were washed twice before analysis using a BD FACS AriaII flow cytometer (San Jose, CA, USA). Lymphocytes were gated on forward and side scatter profiles and analyzed using FlowJo software (Treestar, San Carlos, CA, USA).

The following mAbs were used for cell surface or intracellular staining: FITC-labeled anti-CD45RO (clone: UCHL1), anti-CD69 (clone: FN50), PE-labeled anti-IL-17 (clone: N49-653), anti-T-bet (clone: 4B10), anti-CD25 (clone: 2A3), APC-Cy7-labeled anti-CD4 (clone: GK1.5), APC-labeled anti-CD62L (clone: DREG-56), anti-CCR7 (clone: 3D12), PE-CF594-labeled anti-CD3 (clone: SP342 ), isotype-matched control antibodies, purified anti-CD3, and anti-CD28 mAb which were purchased from BD Bioscience PharMingen. FITC-labeled anti-IL-17 (clone: eBio64DEC17), Pecy7-labeled anti-IFN- $\gamma$ (clone: 4S.B3), Percp-Cy5.5-labeled anti-IL-4 (clone: 8D4-8), APC-labeled
TABle 1: Subjects' characteristics.

\begin{tabular}{lc}
\hline Characteristic & NP \\
\hline Subject numbers & 35 \\
Age (y), mean (range) & $53(43 \sim 72)$ \\
Sex (male/female) & $23 / 12$ \\
Duration (y), mean (range) & $12.5(5-40)$ \\
Atopic & 8 \\
Aspirin intolerance & 0 \\
CT score & $15.5(6 \sim 22)$ \\
Nasal endoscopy score & $4.5(1 \sim 6)$ \\
Mean VAS score & $5.4(3 \sim 8.4)$ \\
\hline
\end{tabular}

anti-IL-22 (clone: IL22JOP), and anti-ROR $\gamma \mathrm{t}$ (clone: AFKJS-9) were purchased from eBioscience (Santiago, Chile).

2.4. Cell Culture Conditions. To analyze the expression of cytokines and transcription factors, mononuclear cells from NP and uncinate tissues were stimulated with PMA (20 $\mathrm{ng} / \mathrm{ml}$; Sigma-Aldrich) and ionomycin ( $1 \mu \mathrm{g} / \mathrm{ml}$; SigmaAldrich) at $37^{\circ} \mathrm{C}$ with $5 \% \mathrm{CO}_{2}$ in the presence of brefeldin A $(10 \mu \mathrm{g} / \mathrm{ml}$; Sigma-Aldrich) for $5 \mathrm{~h}$.

Human nasopharyngeal epithelial cell line NP69 was incubated in complete RPMI-1640 medium at $37^{\circ} \mathrm{C}$ and $5 \% \mathrm{CO}_{2}$. To analyze the responses to virus/bacteria-like stimuli, NP69 cells were seeded in 6 -well plates $\left(2 \times 10^{5}\right.$ cells/well) in the presence or absence of human IFN- $\gamma$ and IL-17A (both at $2 \mathrm{ng} / \mathrm{ml}$, Gibco) for 48 hours. Then, the cells were stimulated with either poly I:C $(10 \mu \mathrm{g} / \mathrm{ml}$; InvivoGen) or LPS (300 ng/ml; Sigma-Aldrich) for $4 \mathrm{~h}$ in serum-free media. Cells cultured in the medium were used as negative controls. Five independent experiments were performed.

2.5. ELISA. IL-17A (eBioscience), IFN- $\gamma$, IL-6, and IL-8 (BD Bioscience PharMingen) production was assayed according to the manufacturer's protocols. The detection limits were as follows: IL-17, $4 \mathrm{pg} / \mathrm{ml}$; IFN- $\gamma, 4.7 \mathrm{pg} / \mathrm{ml}$; IL-6, $1.7 \mathrm{pg} / \mathrm{ml}$; and IL-8, $1.82 \mathrm{pg} / \mathrm{ml}$. For convenient analysis, all values of less than the detectable limit were considered zero.

2.6. ELISpot and Q-PCR. Mononuclear cells from NP and uncinate tissues were stimulated with anti-CD3 and antiCD28 (both $1 \mu \mathrm{g} / \mathrm{ml}$, Sigma-Aldrich) for $24 \mathrm{~h}$. Then, the frequencies of IL-17-producing cells were detected by ELISpot. Spot-forming cells (SFC) were enumerated with the ELISpot image analysis system (Champspot II, Sage Creation, Beijing, China).

The primers used in PCR are shown in Table 2.

2.7. Statistical Analysis. Data are expressed as the mean \pm SEM or median (range). Comparison between two groups was performed by Student's $t$-test. Value of $p<0.05$ (twotailed) was considered significant. 
TABLE 2: Sequence of primers used for qRT-PCR.

\begin{tabular}{lccc}
\hline & \multicolumn{1}{c}{ Forward primer $\left(5^{\prime}-3^{\prime}\right)$} & Reverse primer $\left(5^{\prime}-3^{\prime}\right)$ & Product size $(\mathrm{bp})$ \\
\hline IL-17 & AGATTACTACAACCGATCCACCT & GGGGACAGAGTTCATGTGGTA & 151 \\
IFN- $\gamma$ & TCGGTAACTGACTTGAATGTCCA & TCGCTTCCCTGTTTTAGCTGC & 93 \\
IL-6 & ACTCACCTCTTCAGAACGAATTG & CCATCTTTGGAAGGTTCAGGTTG & 149 \\
IL- 8 & TTTTGCCAAGGAGTGCTAAAGA & AACCCTCTGCACCCAGTTTTC & 194 \\
TNF- $\alpha$ & CCTCTCTCTAATCAGCCCTCTG & GAGGACCTGGGAGTAGATGAG & 220 \\
IL- $1 \beta$ & ATGATGGCTTATTACAGTGGCAA & GTCGGAGATTCGTAGCTGGA & 132 \\
GAPDH & TCAAGAAGGTGGTGAAGCAGG & TCAAAGGTGGAGGAGTGGGT & 115 \\
\hline
\end{tabular}

\section{Results}

3.1. Increased IL-17A in NP Tissues. We collected NP and uncinate samples and found that there were significantly increased protein and mRNA expressions of IL-17A and IFN- $\gamma$ in NP tissues than in uncinate tissues $(p<0.01$, Figures 1(a) and 1(b)). Immunofluorescence histochemical analysis revealed IL-17-producing $\mathrm{CD}^{+}$cells (Figure 1(c)). The ELISpot assay showed that enhanced IL-17A- and IFN$\gamma$-producing cells in the mononuclear cells were induced from NP tissues than uncinate tissues in the addition of anti-CD3 and anti-CD28 $(p<0.05$, Figures $1(\mathrm{~d})$ and $1(\mathrm{e}))$. These data indicated that IL-17A was increased in NP tissues compared to uncinate tissues.

3.2. The Cell Sources of IL-17 Production in NP Tissues. To investigate the source of IL-17A, we performed a single-cell analysis by FACS using lymphocytes isolated from NP tissues and uncinate tissues. The results showed that $\mathrm{CD}^{+} \mathrm{T}$ cells were the major producing cells of IL-17A in NP tissues. And the fraction of IL-17A-expressing $\mathrm{CD}^{+} \mathrm{T}$ cells in NP tissues was significantly higher compared with uncinate tissues $(p<0.01$, Figures 2(a) and 2(b)). Further study demonstrated that IL-17A was produced by $\mathrm{CD}^{+} \mathrm{T}$ cells (Th17), $\mathrm{CD}^{+} \mathrm{T}$ cells (Tc17), and $\gamma \delta \mathrm{T}$ cells $(\gamma \delta \mathrm{T} 17)$ (Figure 2(c)). The percentages of Th17, Tc17, and $\gamma \delta$ T17 cells were increased in NP tissues than in uncinate tissues $(p<0.01$, Figure $2(\mathrm{~d}))$. Among all IL-17A-producing $\mathrm{CD}^{+}$ $\mathrm{T}$ cells, the percentages of $\gamma \delta \mathrm{T} 17$ cells were much more than Th17 and Tc17 cells in NP tissues, suggesting that $\gamma \delta \mathrm{T}$ cells were the most potent IL-17A-producing T cells $(p<0.01$, Figure 2(e)).

3.3. The Majority of Th17 Cells Highly Coexpressed IFN- $\gamma$ in NP Tissues. Then, we determined whether IL-17Aproducing cells coexpressed Th1- and Th2-related cytokines in NP tissues. FACS analysis indicated that CD4 ${ }^{+} \mathrm{IL}-17^{+} \mathrm{T}$ cells expressed higher percentages of IFN- $\gamma$ and IL-22 in NP tissues than uncinate tissues; the expression of IL- 4 by Th17 cells was not significantly different (Figures 3(a) and $3(\mathrm{~b})$ ). IL-17A-producing $\mathrm{CD} 4^{+} \mathrm{T}$ cells mainly coexpressed IL-22 in uncinate tissues. However, the majority of $\mathrm{CD}^{+-}$ IL- $17^{+} \mathrm{T}$ cells coexpressed IFN- $\gamma$ and to a lesser extent IL22 in NP tissues (Figure 3(c)). These data suggested that most of IL-17A ${ }^{+} \mathrm{CD}^{+} \mathrm{T}$ cells were IFN- $\gamma$-producing cells in NP tissues, as Th17/1 cells. More importantly, the percentage of
Th17/1 cells in NP tissues was positively related to endoscopic scores of NP patients (Figure 3(d)).

3.4. The Phenotype of Th17/1 Cells in NP Tissues. We analyzed the basic phenotype of CD $4^{+} \mathrm{IL}-17^{+} \mathrm{IFN}-\gamma^{+} \mathrm{T}$ cells from NP tissues. The results showed that the majority of $\mathrm{CD} 4^{+} \mathrm{IL}-$ $17^{+}$IFN- $\gamma^{+} \mathrm{T}$ cells highly expressed CD45RO, but less of CD62L and CCR7 in NP tissues $(p<0.05$, Figure $4(\mathrm{a}))$, displaying an effector memory phenotype. And more, CD4 ${ }^{+-}$ IL- $17^{+}$IFN- $\gamma^{+}$T cells expressed higher levels of chemokine receptors CXCR3, CXCR4, and CCR6 in NP tissues than in uncinate tissues $(p<0.05$, Figure $4(\mathrm{~b}))$.

Because of the contribution of transcription factors to cytokine expression, we analyzed the expression of $\mathrm{ROR} \gamma \mathrm{t}$ (for IL-17) and T-bet (for IFN- $\gamma$ ) by Th17 cells. We observed that IL-17A ${ }^{+}$cells expressed significantly higher amounts of IFN- $\gamma$, ROR $\gamma$ t, and T-bet than did IL-17 $\mathrm{A}^{-}$cells in NP tissues $(p<0.01$, Figure 5(a)). To further evaluate the regulation of transcription factors, the $\mathrm{CD}^{+} \mathrm{T}$ cells in NP tissues were gated according to the expression of IL-17A and IFN- $\gamma$. The expression of ROR $\gamma \mathrm{t}$ and T-bet was analyzed in each cell subpopulation. Compared to IL-17A IFN- $\gamma^{-} \mathrm{T}$ cells, IL$17 \mathrm{~A}^{+} \mathrm{IFN}-\gamma^{+} \mathrm{T}$ cells highly expressed ROR $\gamma \mathrm{t}$ and T-bet $(p<0.05$, Figure 5(b)). These results demonstrated that Th17/1 in NP tissues not only secreted multiple cytokines but also expressed many transcription factors that should be considered integral to regulating the differentiation and function of nasal polyp-infiltrating lymphocytes.

3.5. The Response of Epithelial Cells to Stimuli in a Th17/1Biased Environment. To investigate the effects of Th17/1 cytokines, human nasopharyngeal epithelial cell NP69 was cultured in the presence of the major Th1 cytokine IFN- $\gamma$ and Th17 cytokine IL-17A. Then, the responses of cells to stimulation with poly I:C or bacterial LPS were analyzed. NP69 cell expression of mRNA for IL-6, IL-8, TNF- $\alpha$, and IL- $1 \beta$ in response to poly I:C was significantly upregulated in Th1/17 cytokine condition when compared to media alone. And poly I:C was the most potent stimulus for increasing the expression of proinflammatory factors. In the meantime, IL- 6 and IL- 8 responses to LPS were also enhanced in a Th1/17 environment, relative to media alone (Figure 6). These data showed that Th17/1-biased environment increased the response of nasal epithelial cells to bacterial and viral-like stimuli. 

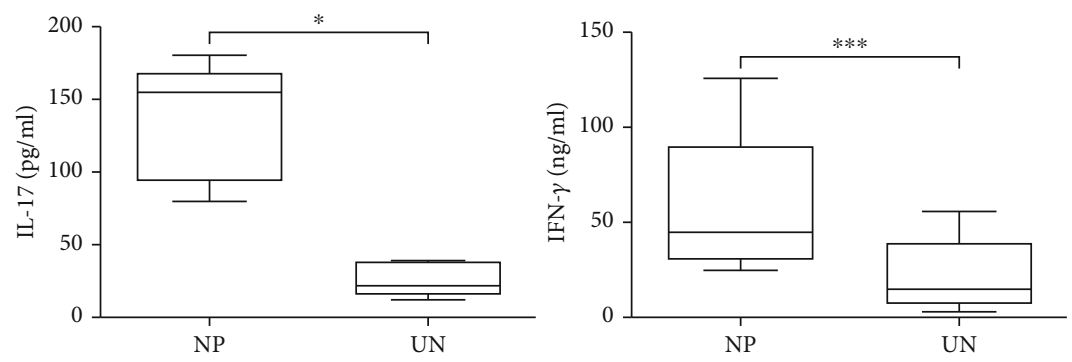

(a)
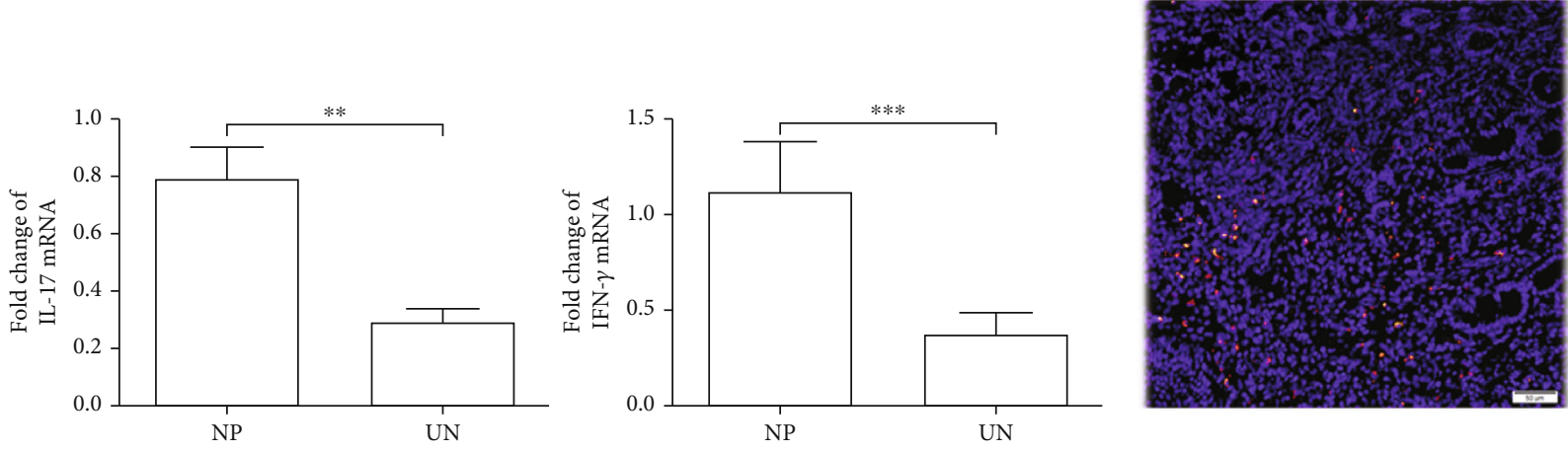

(b)

(c)
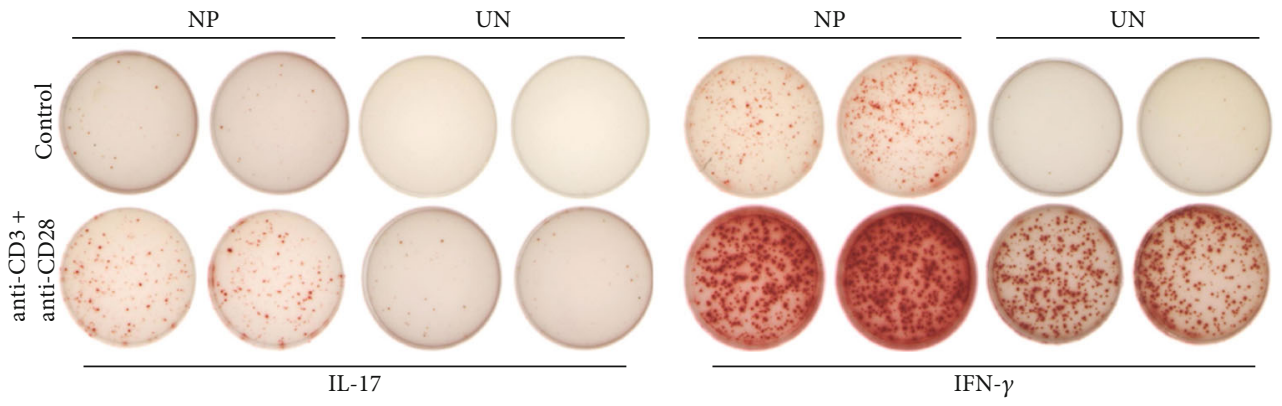

(d)
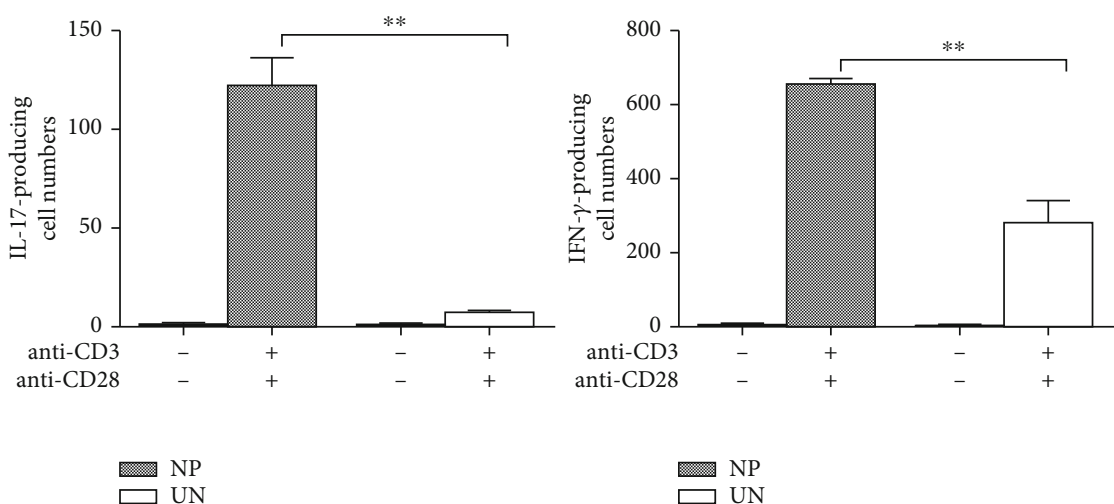

(e)

FIGURE 1: Increased IL-17A in NP tissues. (a) Summary data showed the concentrations of IL-17A and IFN- $\gamma$ in the homogenates of NP $(n=18)$ and uncinate $(n=10)$ tissues. (b) Summary data showed mRNA expression of IL-17A and IFN- $\gamma$ in NP $(n=18)$ and uncinate $(n=10)$ tissues. (c) Representative microphotographs of CD3 (red)-, IL-17 (green)-, and DAPI (blue)-stained section of NP tissues. Scale bars represent $50 \mathrm{mM}$. (d) The mononuclear cells from NP and uncinate tissues were cultured with or without anti-CD3 plus anti-CD28 for $24 \mathrm{~h}$. Representative ELISpot results of IL-17A- and IFN- $\gamma$-producing cells were shown. (e) Summary ELISpot results of IL-17A- and IFN- $\gamma$-producing cells were shown $(n=5)$. Data were shown as the mean \pm SEM. Statistical significance was determined with the MannWhitney test. ${ }^{*} p<0.05 ;{ }^{* *} p<0.01$; ns: no significance; NP: nasal polyp; UN: uncinate. 


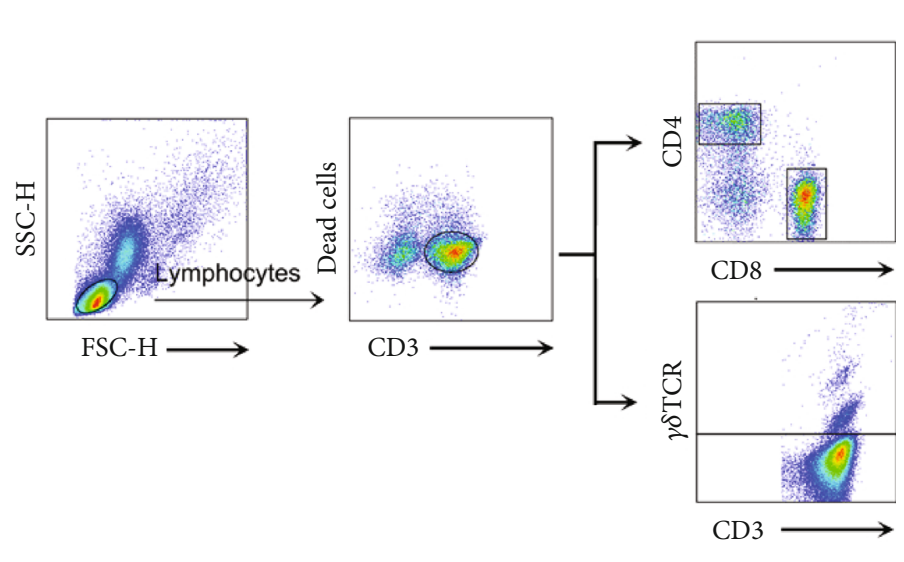

(a)

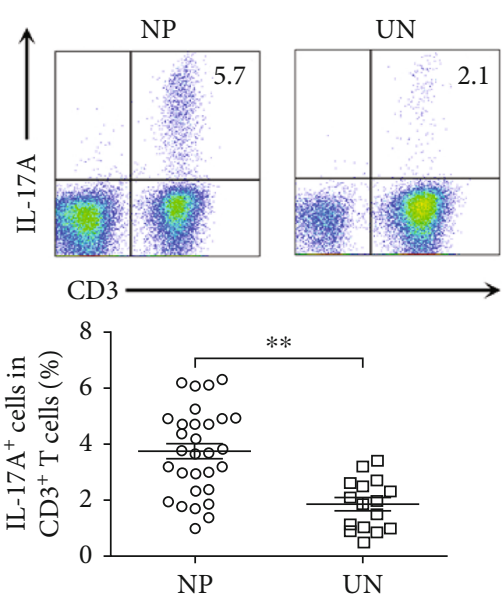

(b)
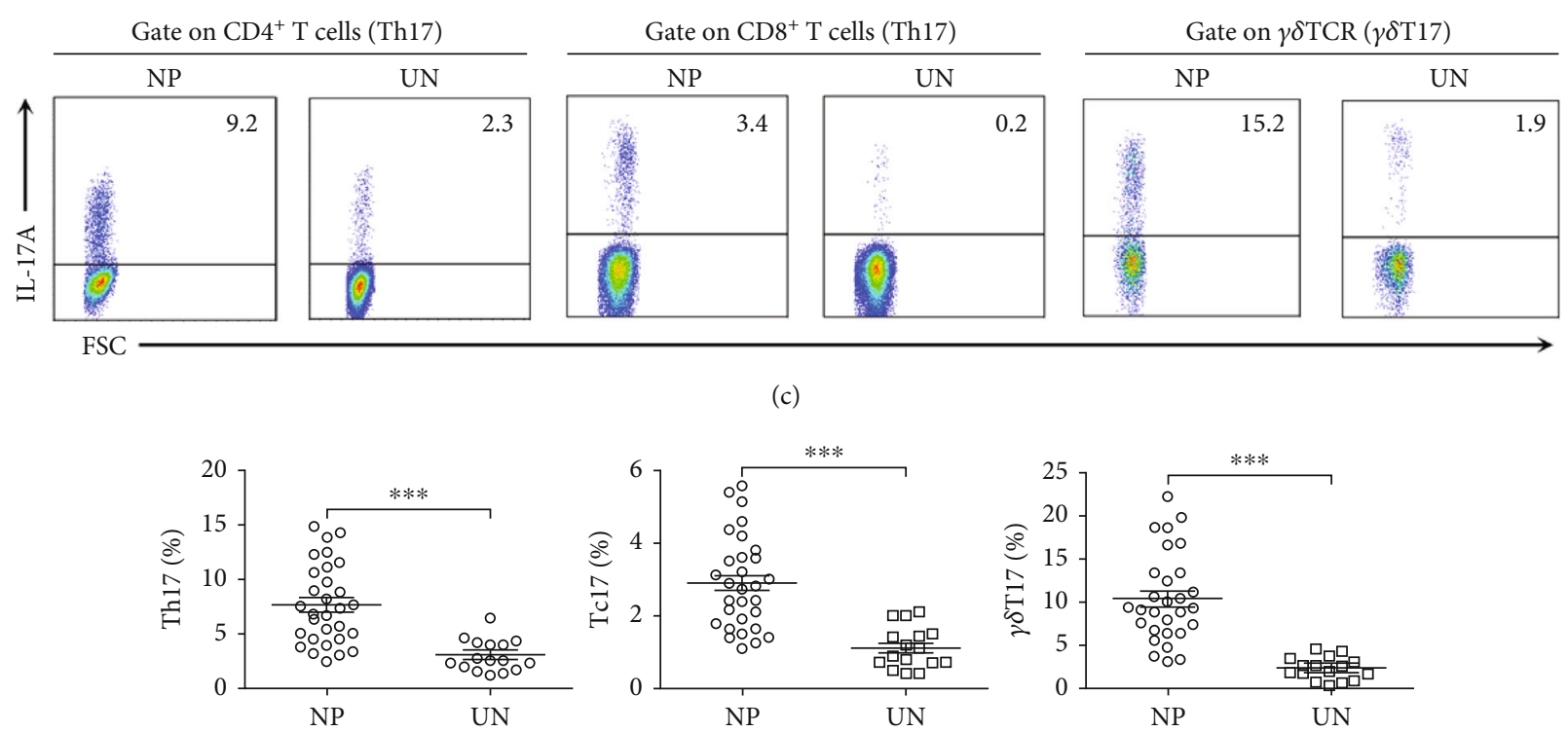

(c)
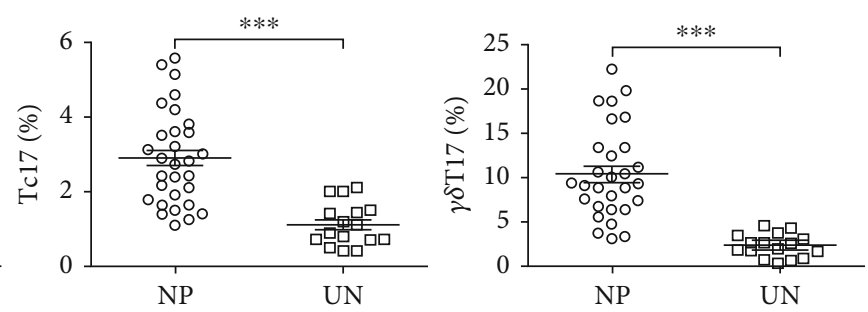

(d)

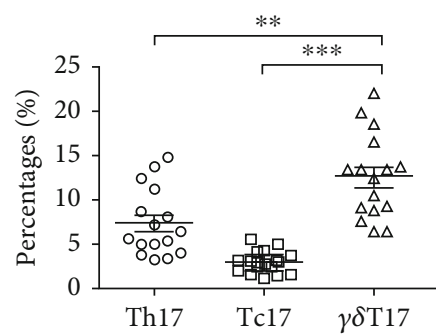

(e)

Figure 2: Both $\mathrm{CD}^{+}, \mathrm{CD}^{+}$, and $\gamma \delta \mathrm{T}$ cells expressed IL-17A in NP tissues. (a) FACS gating was used in the analysis of CD3 ${ }^{+} \mathrm{T}$ cells. (b) Representative FACS graphs and summary data showed the expression of IL-17A by CD3 ${ }^{+} \mathrm{T}$ cells in NP $(n=30)$ and uncinate $(n=16)$ tissues. (c, d) Representative FACS graphs and summary data showed the expression of IL-17A by CD $4^{+}, \mathrm{CD} 8^{+}$, and $\gamma \delta \mathrm{T}$ cells in NP $(n=30)$ and uncinate $(n=16)$ tissues. (e) Summary data showed the percentages of Th17, Tc17, and $\gamma \delta$ T17 cells in NP tissues $(n=16)$. Data were shown as the mean \pm SEM. Statistical significance was determined with the Mann-Whitney test. ${ }^{* *} p<0.01 ;{ }^{* * *} p<0.001$. NP: nasal polyp; UN: uncinate.

\section{Discussion}

IL-17A is a proinflammatory cytokine that plays an essential role in host defense against microbial infections and is impli- cated in various inflammatory conditions. Pathological production of IL-17A leads to excessive inflammatory response and overt tissue damage [10]. Previous reports have showed an enhanced IL-17A expression regardless of eosinophilic 

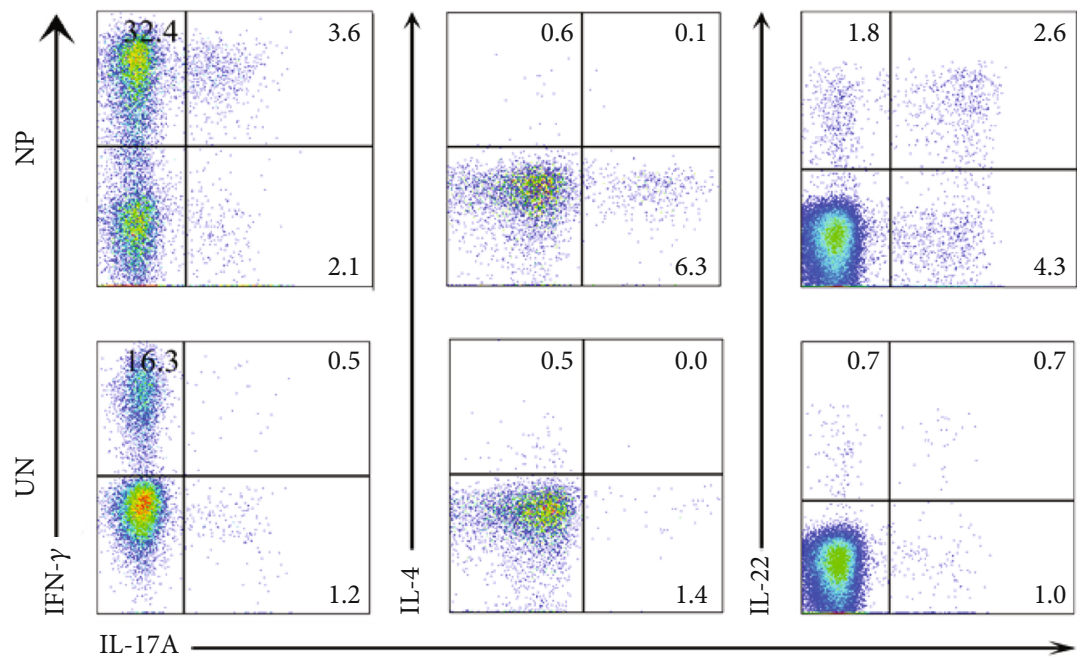

(a)
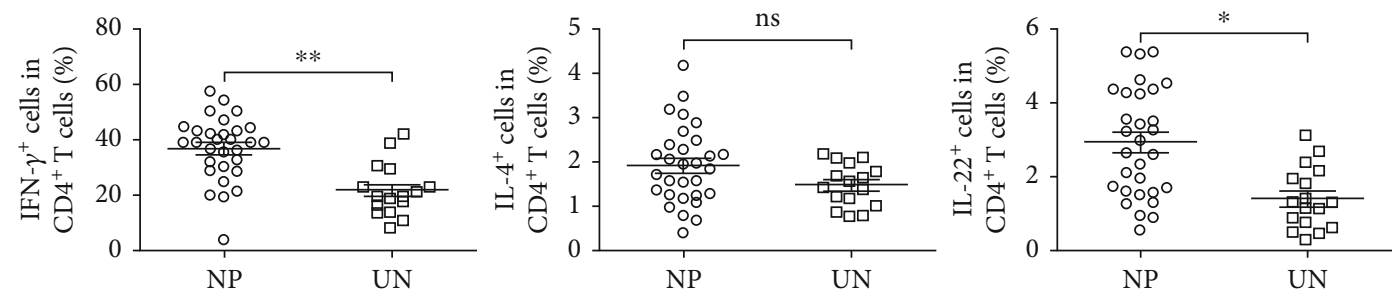

(b)
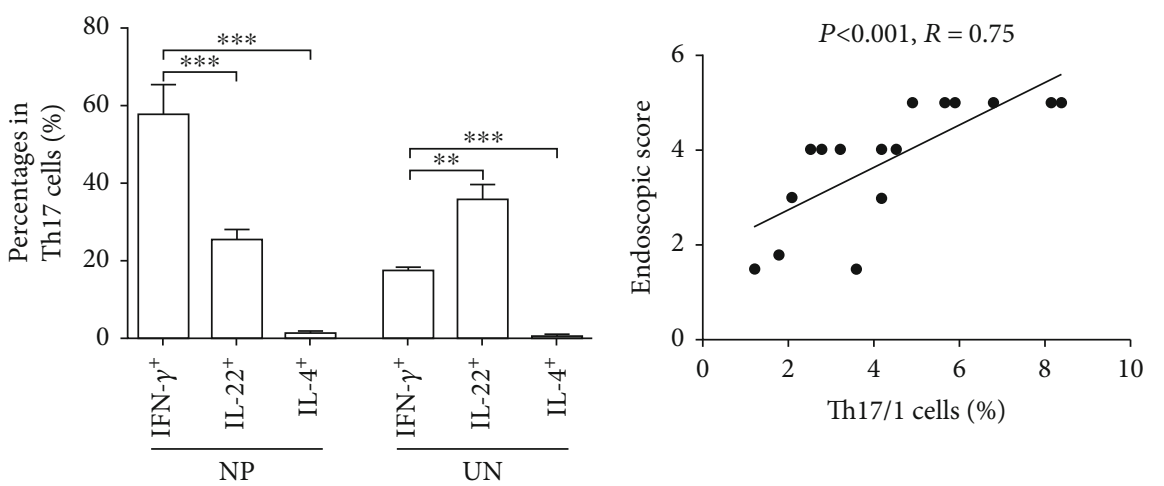

(c)

(d)

Figure 3: The majority of CD4 $4^{+} \mathrm{IL}-17^{+}$cells coexpressed IFN- $\gamma$ cells in NP tissues. $(\mathrm{a}, \mathrm{b})$ Representative graph and summary data showed the expression of IL-17A, IFN- $\gamma$, IL-22, and IL-4 by Th17 cells in NP $(n=30)$ and uncinate $(n=16)$ tissues. (c) Summary data showed the expression of IFN $-\gamma^{+}, \mathrm{IL}-22^{+}$, or IL- $4^{+}$cells in Th17 cells in NP $(n=30)$ and uncinate $(n=16)$ tissues. (d) The percentage of Th17/1 cells in NP tissues was correlated with endoscopic scores $(n=16)$. Correlations were determined by Spearman's rank correlation coefficients. Data were shown as the mean \pm SEM. Statistical significance was determined with the Mann-Whitney test. ${ }^{*} p<0.05$; ${ }^{* *} p<0.01 ;$ ns: no significance; NP: nasal polyp; UN: uncinate.

or noneosinophilic inflammation of CRSwNP [11], despite different results about the role of IL-17A in the severity and development of NPs in the different regions $[12,13]$. The significance of IL-17A in NPs of the Asia area may be more important than that in Europe. Recent studies have shown that IL-17A induced recruitment not only of neutrophils [14] but also of eosinophils in NPs [15]; increased IL-6, IL11 , IL-9, and G-CSF production in human bronchial fibroblasts; and promoted remodeling [16]. In this study, our results also demonstrated that IL-17A was significantly increased in NP tissues than in uncinate tissues. In the upper airway [17], the cellular sources for IL-17A are reported to be T lymphocytes, neutrophils, eosinophils, and epithelial cells. However, the cellular sources of IL-17A are not well understood. We further found that IL-17A was almost exclusively secreted by $\mathrm{CD}^{+} \mathrm{T}$ cells including Th17, Tc17, and $\gamma \delta \mathrm{T} 17$ cells in NP tissues, in which Th17 cells were the major source of IL-17A. Moreover, $\gamma \delta$ T cells were the most potent IL-17A- 

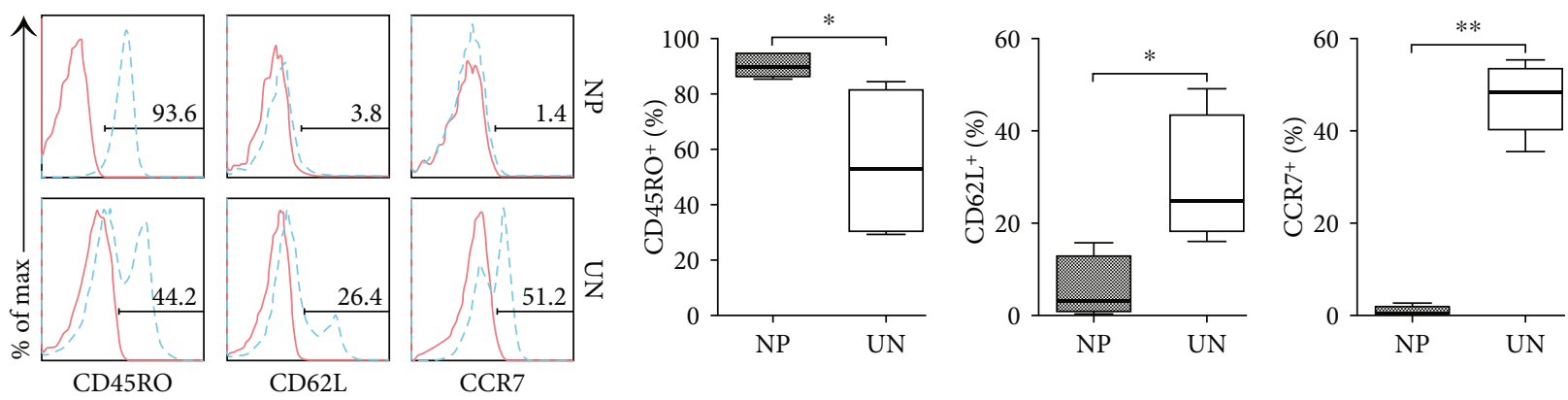

(a)
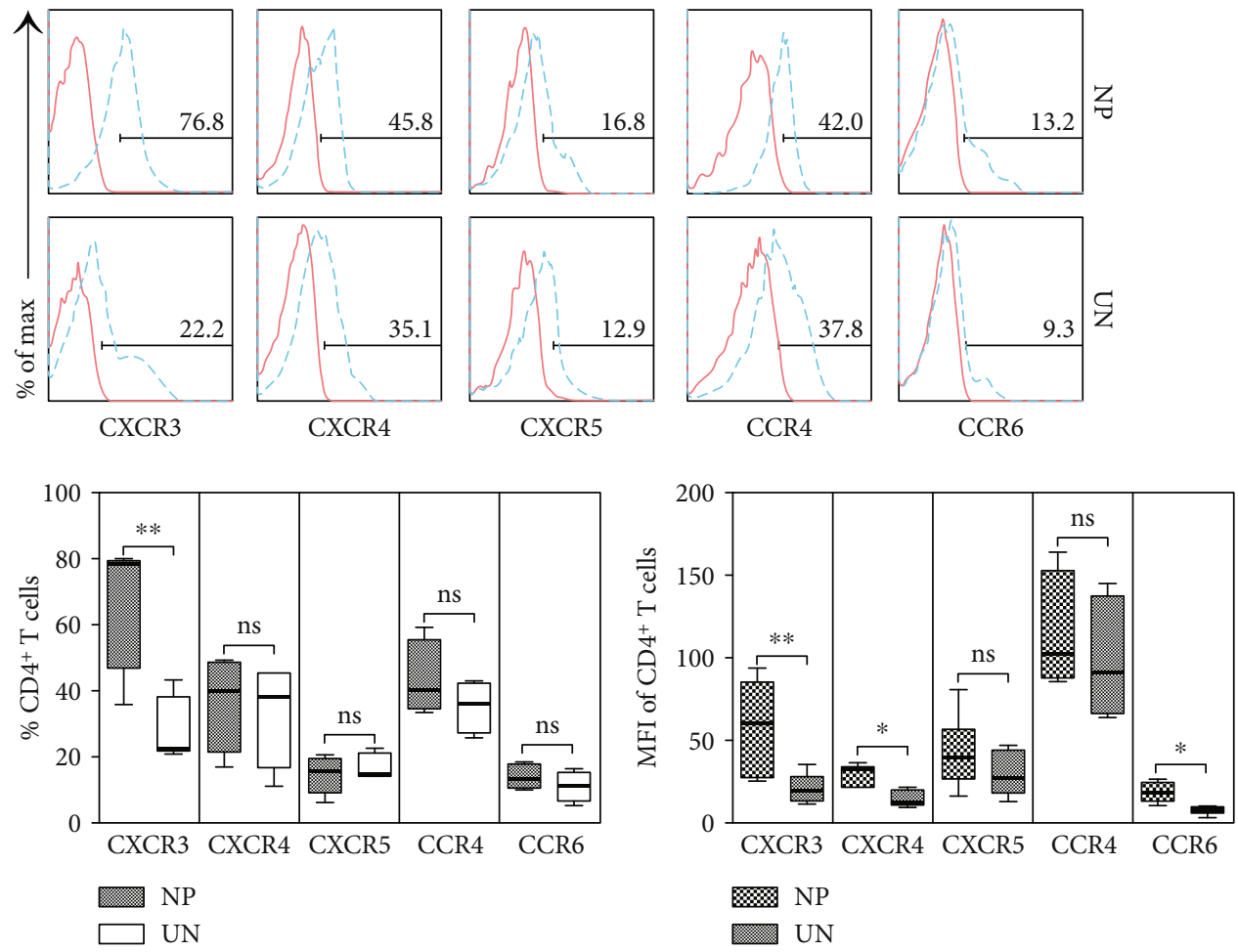

(b)

Figure 4: The phenotype of CD $4^{+} \mathrm{L}-17^{+} \mathrm{IFN}-\gamma^{+}$T cells from NP tissues. (a, b) Representative FACS graphs and summary data showed the expression of CD69 and CD25 on $\mathrm{CD}^{+} \mathrm{T}$ cells of NP $(n=12)$ and uncinate $(n=9)$ tissues. (c, d) Representative FACS graphs and summary data showed the expression of CD45RO, CD62L, and CCR7 on CD3 ${ }^{+}$T cells of NP $(n=12)$ and uncinate $(n=9)$ tissues. Data were shown as the mean \pm SEM. Statistical significance was determined with the Mann-Whitney test. ${ }^{*} p<0.05$; ${ }^{* *} p<0.01$; ns: no significance; NP: nasal polyp; UN: uncinate.

producing cells in NP tissues, though the proportion of $\gamma \delta \mathrm{T}$ cells was less than that of $\mathrm{CD} 4^{+} \mathrm{T}$ and $\mathrm{CD} 8^{+} \mathrm{T}$ cells.

NPs are a common nasal inflammatory disease with regional differences. The pathological features of NPs are characterized by the infiltration of multiple types of inflammatory cells and mixed patterns of $\mathrm{T}$ cell response [18]. According to the European position paper on rhinosinusitis and nasal polyps 2020, it is generally recognized that chronic rhinosinusitis with nasal polyps (CRSwNP) is characterized by infiltration of eosinophils and excessive expression of Th2 cytokines [19]. And recent reports have demonstrated that Chinese or Korean CRSwNP patients possess a distinct pathogenic phenotype including the accumulation of neutrophils and mixed Th1/Th17 response [20, 21]. Many studies reported that Th17 cells expressed both IL-17A and IFN- $\gamma$ at the same time in some of the pathological conditions, indicating a high degree of developmental flexibility [22]. The developmental flexibility of Th17 cells and their shift to a Th1 cell-like phenotype has been linked to the pathogenicity of Th17 cells in infectious diseases [23], autoimmune diseases [24], and tumors [25]. We analyzed the cytokine expression of Th17 cells in NP tissues using flow cytometry. The results showed that the majority of IL-17A-expressing $\mathrm{CD} 4{ }^{+} \mathrm{T}$ cells coexpressed IFN- $\gamma$, not IL- 4 or IL-22, and there was a significant increase of Th17/1 cells in NP tissues.

Published studies [26] have described that $\mathrm{CD}^{+} \mathrm{T}$ cells simultaneously produced IL-17A, and IFN- $\gamma$ stably coexpressed Th17 and Th1 cell master transcription factors 

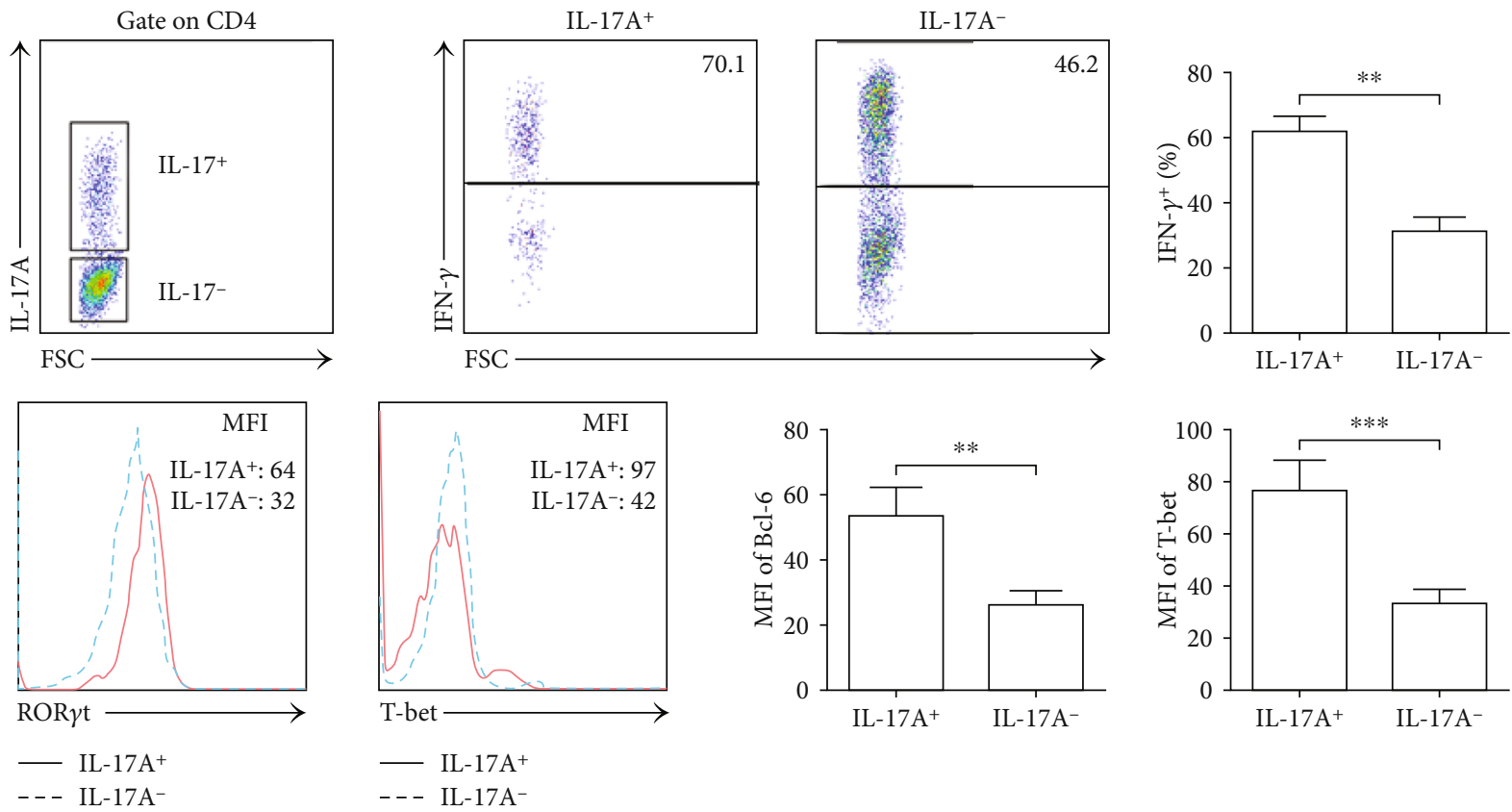

(a)
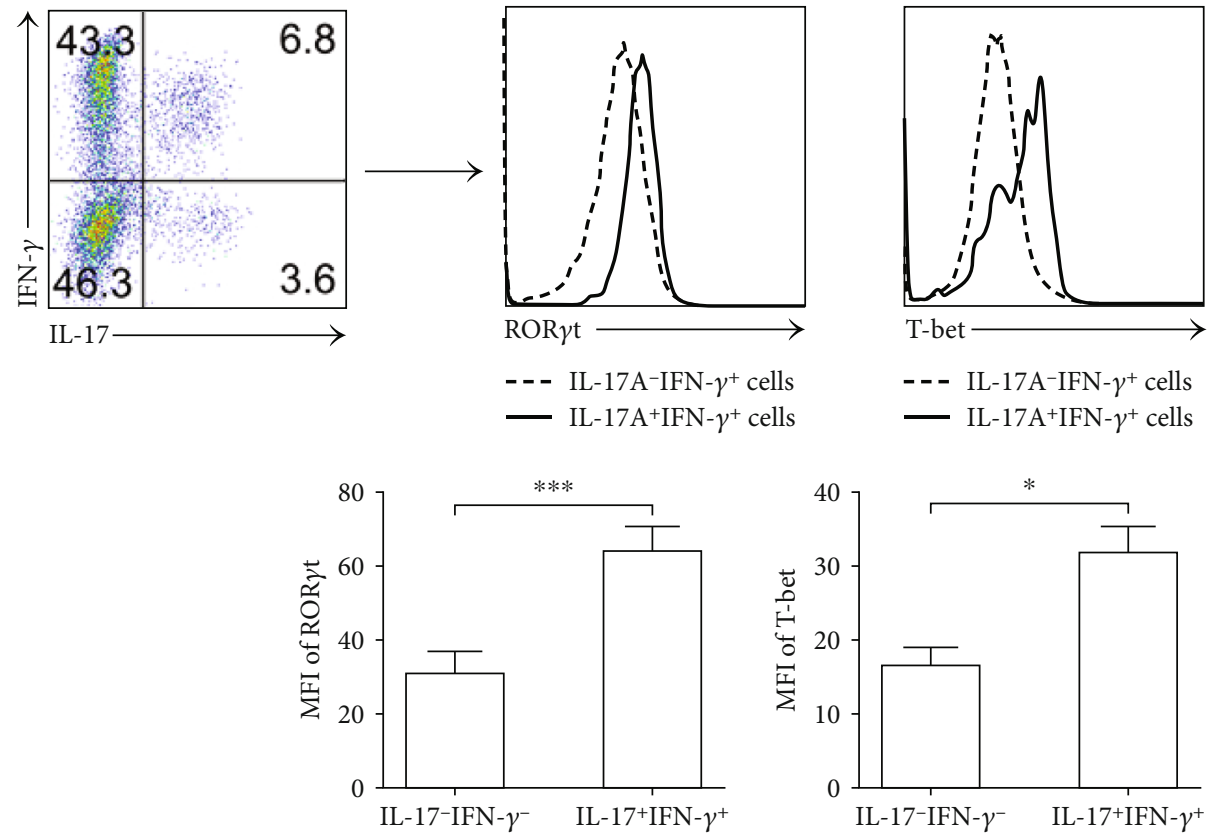

(b)

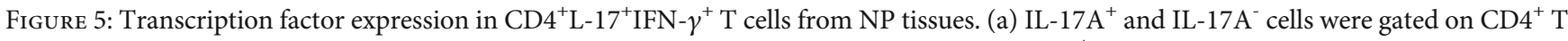
cells in NP tissues. The expression of IFN- $\gamma, \operatorname{ROR} \gamma \mathrm{t}$, and T-bet was analyzed $(n=12)$. (b) $\mathrm{CD}^{+}{ }^{+} \mathrm{T}$ cells from NP tissues were gated on the expression of IL-17A and IFN- $\gamma$. Representative FACS and summary data showed the expression of ROR $\gamma$ t, T-bet in IL-17 IFN- $\gamma^{+}$, and IL-17 IFN- $\gamma^{-}$cells $(n=12)$. Data were shown as the mean \pm SEM. Statistical significance was determined with the Mann-Whitney test. ${ }^{*} p<0.05 ;{ }^{* *} p<0.01 ;{ }^{* * *} p<0.001$.

ROR $\gamma \mathrm{t}$ and T-bet. However, the coexpression of ROR $\gamma \mathrm{t}$ and T-bet was not sufficient to generate Th cells with dual Th17 and Th1 cell features. The development of IL-17A IFN- $\gamma^{+}$ or Th1-like Th17 cells was dependent on T-bet, Runx1, and Runx3 [27]. Our results demonstrated that $\mathrm{CD} 4^{+} \mathrm{IL}-$ $17 \mathrm{~A}^{+}$IFN $-\gamma^{+} \mathrm{T}$ cell highly expressed CXCR3 and CCR6, T- bet, and ROR $\gamma t$ in NP tissues. However, the precise function of IL-17A $\mathrm{A}^{+} \mathrm{IFN}-\gamma^{+}$cells in NP tissues needs further study.

In humans, IFN- $\gamma$-producing Th17 cells (Th17/1 cells), identified by coexpression of the chemokine receptors CCR6 and CXCR3, have been proposed to be highly pathogenic functions due in part to their expression of the 

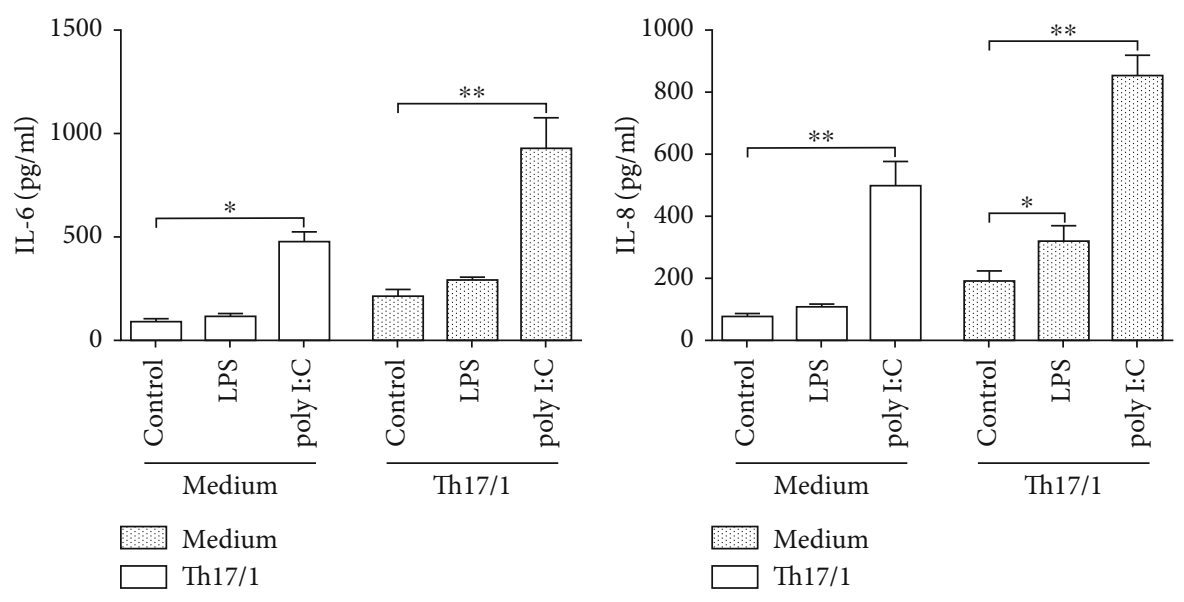

(a)
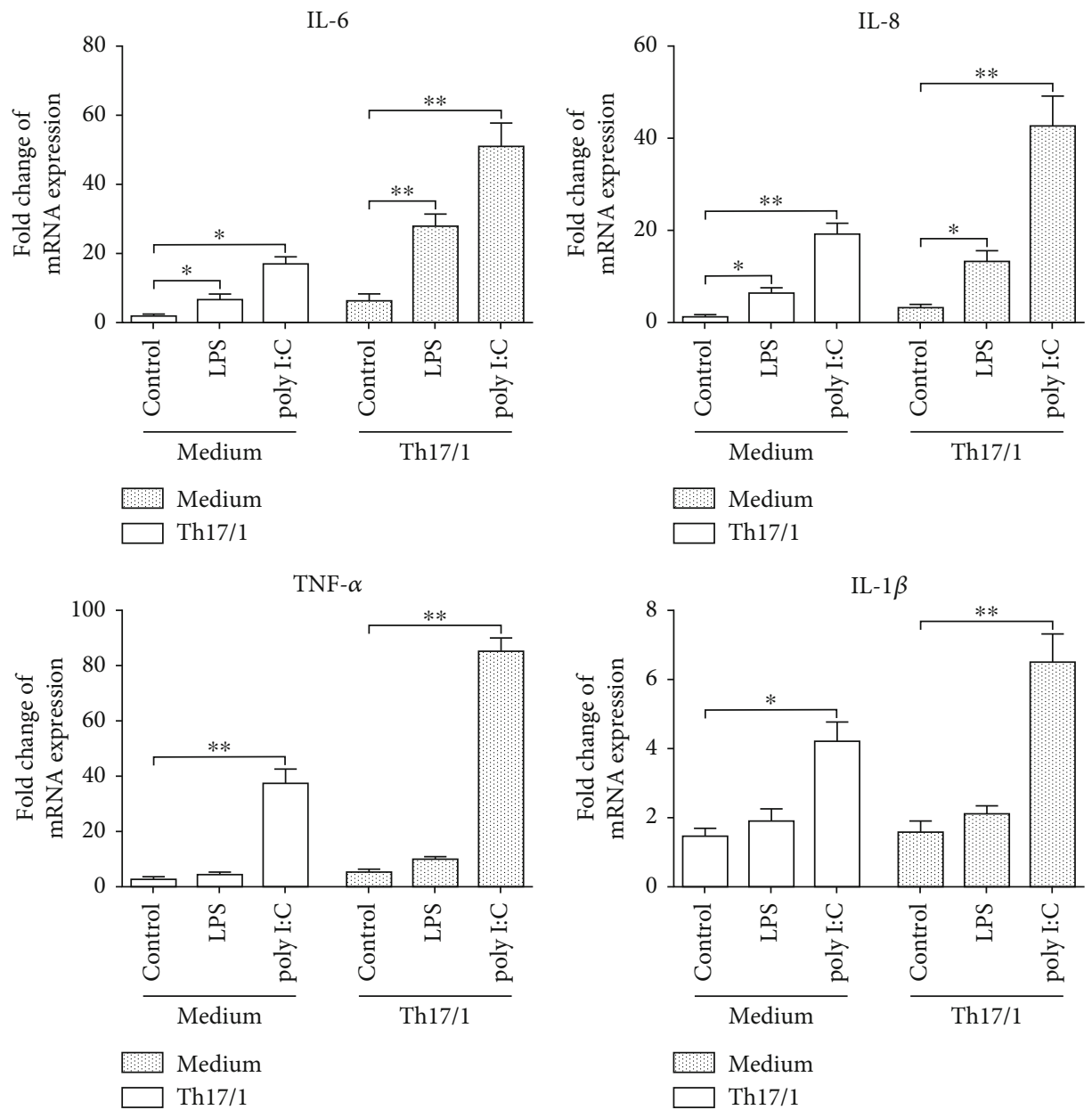

(b)

FIGURE 6: Effect of a Th17/1 environment on the proinflammatory response of NP69 cell to stimuli. NP69 cells were cultured in the presence or absence of human IFN- $\gamma$ and IL-17A for 48 hours, then stimulated with either poly I:C or LPS for $4 \mathrm{~h}$ in serum-free media. (a) Summary data showed IL-6 and IL-8 expression levels by NP69 cells $(n=5)$. (b) Summary data showed mRNA expression of IL-6, IL-8, TNF- $\alpha$, and IL$1 \beta$ in NP69 cells $(n=5)$. Data were shown as the mean \pm SEM. Statistical significance was determined with the Mann-Whitney test. ${ }^{*} p<0.05$; ${ }^{* *} p<0.01 ;{ }^{* * *} p<0.001$. 
proinflammatory cytokines IL-17, IFN- $\gamma$, and GM-CSF [28]. Reduction of Th1-like Th17 cell numbers is a potential strategy for the treatment of autoimmune diseases [29]. The acquisition of Th1 effector cell functions by Th17 cells, including IFN- $\gamma$ expression, is crucial for the effective antitumor activity of Th17 cells [30]. However, their developmental requirements, relationship with classical Th17 and Th1 cells, and physiological role in normal immune responses are not well understood. We found that Th17/1-biased environment increased the response of nasal epithelial cells to bacterial and viral stimuli, implying that Th1-like Th17 cells play a greater role in the pathological development of NPs than Th17 or Th1 cells.

\section{Data Availability}

The data that support the findings of this study are available from the corresponding author upon reasonable request.

\section{Conflicts of Interest}

The authors declare no competing financial interests.

\section{Acknowledgments}

This work was supported by the National Natural Science Foundation of China (Grant No. 81903916).

\section{References}

[1] T. E. Del and J. Portela, "Nasal polyps," 2020.

[2] C. Bachert, N. Zhang, C. Cavaliere, W. Weiping, E. Gevaert, and O. Krysko, "Biologics for chronic rhinosinusitis with nasal polyps," The Journal of Allergy and Clinical Immunology, vol. 145, no. 3, pp. 725-739, 2020.

[3] T. M. Laidlaw and K. M. Buchheit, "Biologics in chronic rhinosinusitis with nasal polyposis," Annals of Allergy, Asthma \& Immunology, vol. 124, no. 4, pp. 326-332, 2020.

[4] L. Xiao, L. Jia, Y. Zhang et al., "Human IL-21+IFN- $\gamma+$ CD4+ T cells in nasal polyps are regulated by IL-12," Scientific Reports, vol. 5, no. 1, p. 12781, 2015.

[5] Z. Zhu, W. Wang, X. Zhang et al., "Nasal fluid cytology and cytokine profiles of eosinophilic and non-eosinophilic chronic rhinosinusitis with nasal polyps," Rhinology, vol. 58, no. 4, pp. 314-322, 2020.

[6] K. König, C. Klemens, M. Haack et al., "Cytokine patterns in nasal secretion of non-atopic patients distinguish between chronic rhinosinusitis with or without nasal polys," Allergy, Asthma and Clinical Immunology, vol. 12, no. 1, p. 19, 2016.

[7] M. Wang, N. Zhang, M. Zheng et al., "Cross-talk between TH2 and TH17 pathways in patients with chronic rhinosinusitis with nasal polyps," The Journal of Allergy and Clinical Immunology, vol. 144, no. 5, pp. 1254-1264, 2019.

[8] X. Chen, L. Chang, X. Li et al., "Tc17/IL-17A up-regulated the expression of MMP-9 via NF- $\kappa$ B pathway in nasal epithelial cells of patients with chronic rhinosinusitis," Frontiers in Immunology, vol. 9, p. 2121, 2018.

[9] W. Fokkens, V. Lund, and J. Mullol, “EP3OS 2007: European position paper on rhinosinusitis and nasal polyps 2007. A summary for otorhinolaryngologists," Rhinology, vol. 45, no. 2, pp. 97-101, 2007.
[10] M. J. McGeachy, D. J. Cua, and S. L. Gaffen, "The IL-17 family of cytokines in health and disease," Immunity, vol. 50, no. 4, pp. 892-906, 2019.

[11] X. D. Jiang, G. Y. Li, L. Li, Z. Dong, and D. D. Zhu, "The characterization of IL-17A expression in patients with chronic rhinosinusitis with nasal polyps," American Journal of Rhinology \& Allergy, vol. 25, no. 5, pp. e171-e175, 2011.

[12] S. L. Hong, Y. L. Zhang, S. W. Kim et al., "Interleukin-17Ainduced inflammation does not influence the development of nasal polyps in murine model," International forum of allergy \& rhinology, vol. 5, no. 5, pp. 363-370, 2015.

[13] N. Van Bruaene, C. A. Pérez-Novo, T. M. Basinski et al., “Tcell regulation in chronic paranasal sinus disease," The Journal of Allergy and Clinical Immunology, vol. 121, no. 6, pp. 14351441.e3, 2008.

[14] S. Yu, C. Cao, Q. Li et al., "Local IL-17 positive T cells are functionally associated with neutrophil infiltration and their development is regulated by mucosal microenvironment in nasal polyps," Inflammation Research, vol. 70, no. 1, pp. 139-149, 2021.

[15] C. Sun, H. Ouyang, and R. Luo, "Distinct characteristics of nasal polyps with and without eosinophilia," Brazilian Journal of Otorhinolaryngology, vol. 83, no. 1, pp. 66-72, 2017.

[16] H. Homma, K. Kamiya, T. Kusunoki, and K. Ikeda, "Multiplex analyses of cytokine and chemokine release from the cultured fibroblast of nasal polyps: the effect of IL-17A," Acta Oto-Laryngologica, vol. 133, no. 10, pp. 1065-1072, 2013.

[17] D. Bullens, A. Decraene, S. Seys, and L. J. Dupont, "IL-17A in human respiratory diseases: innate or adaptive immunity? Clinical implications," Clinical and Developmental Immunology, vol. 2013, Article ID 840315, 8 pages, 2013.

[18] X. Lin, X. Zhuang, C. Li, and X. Wang, "Interactions between dendritic cells and T lymphocytes in pathogenesis of nasal polyps," Experimental and Therapeutic Medicine, vol. 15, no. 6, pp. 5167-5172, 2018.

[19] W. J. Fokkens, V. J. Lund, C. Hopkins et al., "European position paper on rhinosinusitis and nasal polyps 2020," Rhinology, vol. 58, Suppl S29, pp. 1-464, 2020.

[20] Q. L. Zhao, L. Yu, L. L. Zhi et al., "The relationship between lung function and the clinical and histopathological features in Chinese patients with nasal polyps," The Journal of Laryngology and Otology, vol. 131, no. 10, pp. 880-888, 2017.

[21] W. Wang, Y. Gao, Z. Zhu et al., "Changes in the clinical and histological characteristics of Chinese chronic rhinosinusitis with nasal polyps over 11 years," International forum of allergy \& rhinology, vol. 9, no. 2, pp. 149-157, 2019.

[22] H. Xu, T. Agalioti, J. Zhao et al., "The induction and function of the anti-inflammatory fate of TH17 cells," Nature Communications, vol. 11, no. 1, p. 3334, 2020.

[23] J. Li, M. Hua, X. Hu et al., "Dexamethasone suppresses the Th17/1 cell polarization in the CD4(+) T cells from patients with primary immune thrombocytopenia," Thrombosis Research, vol. 190, pp. 26-34, 2020.

[24] T. Moser, K. Akgün, U. Proschmann, J. Sellner, and T. Ziemssen, "The role of TH17 cells in multiple sclerosis: therapeutic implications," Autoimmunity Reviews, vol. 19, no. 10, p. 102647, 2020.

[25] P. Gaur, N. K. Shukla, and S. N. Das, "Phenotypic and functional characteristics of Th17 (CD4(+)IL17A(+)) cells in human oral squamous cell carcinoma and its clinical 
relevance," Immunological Investigations, vol. 46, no. 7 , pp. 689-702, 2017.

[26] J. Leipe, F. Pirronello, A. Klose, H. Schulze-Koops, and A. Skapenko, "Increased plasticity of non-classic Th1 cells toward the Th17 phenotype," Modern Rheumatology, vol. 30, no. 5, pp. 930-936, 2020.

[27] Y. Wang, J. Godec, K. Ben-Aissa et al., "The transcription factors T-bet and Runx are required for the ontogeny of pathogenic interferon- $\gamma$-producing $\mathrm{T}$ helper 17 cells," Immunity, vol. 40, no. 3, pp. 355-366, 2014.

[28] S. Fang, S. Zhang, Y. Huang et al., "Evidence for associations between Th1/Th17 "hybrid" phenotype and altered lipometabolism in very severe Graves orbitopathy," The Journal of Clinical Endocrinology and Metabolism, vol. 105, no. 6, pp. 1851-1867, 2020.

[29] A. N. Kamali, S. M. Noorbakhsh, H. Hamedifar et al., "A role for Th1-like Th17 cells in the pathogenesis of inflammatory and autoimmune disorders," Molecular Immunology, vol. 105, pp. 107-115, 2019.

[30] Z. Asadzadeh, H. Mohammadi, E. Safarzadeh et al., "The paradox of Th17 cell functions in tumor immunity," Cellular Immunology, vol. 322, pp. 15-25, 2017. 